\title{
ON THE MOTION OF INCOMPRESSIBLE VISCOUS FLUID WITH FLOTATION ON FREE SURFACE
}

\author{
TRAN THU HA \\ Institute of Mechanics NCSR Vietnam
}

\begin{abstract}
In the works $[1,15]$ the problems about the mations of ideal fluid contained in an elastic bottom vessel or in a solid vessel with flotations on the free surfaces were investigated. In this paper we consider the motion of incompressible viscous fluid contained in a vessel with flotation on the free surface. The unique existence theorem of solution is proved, the spectrurn structure and the completeness of the characteristic vector system are studied.
\end{abstract}

\section{FORMULATION ON THE PROBLEM}

Let us introduce an incompressible viscous fluid contained in a vessel. On the solid boundary $S$ the boundary condition $v=0$ where $v=\left(v_{1}, v_{2}, v_{3}\right)$ is the velocity of the fluid particles. As in the work [1] it is supposed that the weighable particles of some substances float on the free surface $\Gamma$. On the free vibration process they are not acting each with other or their interaction is negligible. From the dynamical of view, the availability of the no-interacting one another particles floating on the free fluid surface $\Gamma$ may be interpreted when the free surface is regarded as weighable surface with surface density of mass distribution $\delta(x)(x \in \Gamma)$ where $\delta(x) \geq 0$ and may become zero indentically in some subregions of the region $\Gamma$.

We consider the elementary conclusion of the boundary condition for the free weighable surface $\Gamma$. As in the works $[1,2,3]$ we write the second Newton law for the element $\Delta \Gamma$ of the free surface at the point $x \in \Gamma$. In the linear approximation this law is written in the following form

$$
\begin{gathered}
\Delta \Gamma \delta(x) \frac{\partial v_{3}}{\partial t}=\Delta \Gamma\left[p-2 \rho \nu \frac{\partial v_{3}}{\partial x_{3}}-\rho g \xi\right] \quad \text { on } \Gamma \\
\tau_{i 3}(v)=\frac{\partial v_{i}}{\partial x_{3}}+\frac{\partial v_{3}}{\partial x_{i}}=0, \quad i=1,2 \text { on } \Gamma
\end{gathered}
$$

Here $p$ is the Huid pressure, $\rho$ - the fluid density, $\nu$ - the coefficient of kinematic viscousity.

Taking $v_{3}=\partial \xi / \partial t$ into consideration in the equation (1.1) we get

$$
\frac{\partial}{\partial t}\left[p-2 \rho \nu \frac{\partial v_{3}}{\partial x_{3}}\right]=\rho g v_{3}+\delta(x) \frac{\partial^{2} v_{3}}{\partial t^{2}}
$$

If $\delta(x)=0$, the condition (1.2) become the condition of the problem in the work [4].

As in the works $[3,4]$ we get the boundary problem describing the motion of the fiuid:

$$
\begin{array}{ll}
\frac{\partial v}{\partial t}=-\frac{1}{\rho} \nabla p+\nu \Delta v+\frac{1}{\rho} f & \text { in } \Omega \\
\operatorname{div} v=0 & \text { in } \Omega \\
v=0 & \text { on } S
\end{array}
$$




$$
\begin{array}{cc}
\frac{\partial}{\partial t}\left[p-2 \rho \nu \frac{\partial v_{3}}{\partial x_{3}}\right]=\rho g v_{3}+\delta(x) \frac{\partial^{2} v_{3}}{\partial t^{2}} & \text { on } \Gamma \\
\tau_{13}(v)=\tau_{23}(v)=0 & \text { on } \Gamma \\
p(x, 0)=p^{0}(x), \quad v(x, 0)=v^{0}(x), & \left.\frac{\partial v(x, 0)}{\partial t}\right|_{t=0}=v^{1}(x)
\end{array}
$$

\section{FUNCTION SPACES AND AUXILIARY PROBLEMS}

Denote $L_{2}(\Omega)$ by the space of quadratically integrable vector-functions $v$ in $\Omega$. In [11] the following decomposition of $L_{2}(\Omega)$ is given

$$
L_{2}(\Omega)=\widetilde{L}_{2}(\Omega) \oplus G(\Omega)
$$

where

$$
\begin{aligned}
\tilde{L}_{2}(\Omega) & =\left\{\begin{array}{lll}
v: v \in L_{2}(\Omega), & \operatorname{div} v=0, \quad v_{n}=0 \text { on } S
\end{array}\right. \\
G(\Omega) & =\left\{\begin{array}{ll}
v: v \in L_{2}(\Omega), & v=\nabla p, \quad p=0 \text { on } \Gamma
\end{array}\right\}
\end{aligned}
$$

In [3] the Veill's decomposition of $\tilde{L}_{2}(\Omega)$ is given as follows:

$$
\tilde{L}_{2}(\Omega)=J_{1}(\Omega) \oplus J_{0}(\Omega)
$$

where

$$
\begin{aligned}
& J_{1}(\Omega)=\left\{v: v \in \tilde{L}_{2}(\Omega), \quad v=\nabla_{p}\right\} \\
& J_{0}(\Omega)=\left\{u: u \in \tilde{L}_{2}(\Omega), \quad u \cdot \bar{n}=0 \text { on } \Gamma\right\}
\end{aligned}
$$

Here $\bar{n}$ is the external normal of the free surface $\Gamma$.

Let us define $W_{2,0}^{1}(\Omega)$ as the space of vector functions $u \in \tilde{L}_{2}(\Omega)$ getting generalized derivaties of the first order and vanishing out - side some compact subsets of $S$. Their scalar product in $W_{2,0}^{1}(\Omega)$ is defined as follows

$$
(u, v)_{W_{3,0}^{1}(\Omega)}=\sum_{i=1}^{3} \int_{\Omega} \nabla u_{x_{i}} \nabla v_{x_{i}} d \Omega
$$

so

$$
\|u\|_{W_{1,0}^{1}(\Omega)}=\left[(u, u)_{W_{2,0}^{1}(\Omega)}\right]^{1 / 2}
$$

In [3] the decomposition of $W_{2,0}^{1}(\Omega)$ is given as follows:

$$
W_{2,0}^{1}(\Omega)=\tilde{W}_{2}^{1}(\Omega) \oplus U_{2}^{1}(\Omega)
$$

where

$$
\begin{aligned}
& \tilde{W}_{2}^{1}(\Omega)=\left\{u ; 0^{u} \in W_{2,0}^{1}(\Omega), \quad u_{3}=0 \text { on } \Gamma\right\} \\
& U_{2}^{1}(\Omega)=\left\{v: v \in W_{2,0}^{1}(\Omega), \quad v=\nabla p\right\}
\end{aligned}
$$


Denote $H_{2}(\Gamma)$ by the space of quadratically integrable functions $\varphi \in \Gamma$. Let $H_{2}^{-1 / 2}(\Gamma)$ be the duel space of $H_{2}^{1 / 2}(\Gamma)[12]$. We define the space $H_{0}, H_{+}, H_{-}$as follows:

$$
H_{0}=H_{2}(\Gamma) \ominus\{1\}, \quad H_{+}=H_{0} \cap H_{2}^{1 / 2}, \quad H_{-}=H_{0} \cap H_{2}^{-1 / 2}(\Gamma) .
$$

Problem 1. Let there be given a vector-function $h \in \tilde{L}_{2}(\Omega)$ we seek a vector-function $v^{(1)}$ and a function $p^{(1)}$ so that the following equations and conditions are satisfied:

$$
\begin{array}{ll}
-\nu \Delta v^{(1)}+\frac{1}{\rho} \nabla p^{(1)}=h, \quad \operatorname{div} v^{(1)}=0 ; & \text { in } \Omega \\
v^{(1)}=0 ; & \text { on } S \\
p^{(1)}-2 \rho \nu \frac{\partial v_{3}^{(1)}}{\partial x_{3}}=0 ; \quad \tau_{i 3}\left(v^{(1)}\right)=0(i=1,2) & \text { on } \Gamma
\end{array}
$$

Problem 2. Let there be given a function $\Psi \in H_{\text {- }}$ we seek a vector-function $v^{(2)}$ and a function $p^{(2)}$ so that the following equations and conditions are satisfreds

$$
\begin{array}{ll}
-\nu \Delta v^{(2)}+\frac{1}{\rho} \nabla p^{(2)}=0, \quad \operatorname{div} v^{(2)}=0 ; & \text { in } \Omega \\
v^{(2)}=0 ; & \text { on } S \\
p^{(2)}-2 \rho \nu \frac{\partial v_{3}^{(2)}}{\partial x_{3}}=\Psi ; \quad \tau_{i 3}\left(v^{(2)}\right)=0(i=1,2) & \text { on } \Gamma
\end{array}
$$

In [4] we find the following lemmas:

Lemma 1. For the vector function $h \in \tilde{L}_{2}(\Omega)$ there exists the unique generalized solution of the problem 1 . This solution is the vector-function $v^{(1)}$ satisfying the equality:

$$
\left(v^{(1)}, v\right)_{W_{2,0}^{1}(\Omega)}=(h, v)_{\tilde{L}_{2}(\Omega)}, \quad \forall v \in W_{2,0}^{1}(\Omega)
$$

The operator $A_{1}^{-1}$ giving the solution of the problem $\left(v^{(1)}=A_{1}^{-1} h\right)$ is positive and compact, it $\operatorname{maps} \tilde{L}_{2}(\Omega)$ into $W_{2,0}^{1}(\Omega)$ and $D\left(A_{1}^{1 / 2}\right)=W_{2,0}^{1}(\Omega), A_{1}^{1 / 2} W_{2,0}^{1}(\Omega)=\tilde{L}_{2}(\Omega)$.

In [4] it was proved that $A_{1}^{1 / 2} \tilde{W}_{2}^{1}(\Omega)=J_{0}(\Omega)$ and $A_{1}^{1 / 2} U_{2}^{1}(\Omega)=J_{1}(\Omega)$

Lemma 2. For the function $\Psi \in H_{-}$there exists the unique generalized solution of the problem 2. This solution is the vector-function $v^{(2)}$ satisfying the equality:

$$
\left(v^{(2)}, s\right)_{W_{2,0}^{1}(0)}=(\Psi, \gamma s)_{H_{0}},\left.\quad \gamma s \equiv s_{3}\right|_{\Gamma}
$$

The operator $Q$ giving the solution of the problem $\left(v^{(2)}=Q \Psi\right)$ is compact and it maps $H_{-}$into $W_{2,0}^{1}(\Omega)$.

\section{THE UNIQUE EXISTENCE THEOREM OF THE SOLUTION}

Using the lemmas 1,2 we can prove that the equations (1.3), (1.4) with the conditions (1.5) - (1.7) are equivalent to the following equation system 


$$
\begin{aligned}
& v=-\nu^{-1} \frac{d}{d t} A_{1}^{-1} v+\nu^{-1} A_{1}^{-1} f \\
& -\frac{d \varphi}{d t}=\rho g \gamma v+\delta(x) \frac{d^{2}}{d t^{2}} \gamma^{v}
\end{aligned}
$$

Hеге $\varphi=-p+2 \nu \rho \partial v_{3} / \partial x_{3}$ and the operator $\gamma: W_{2,0}^{1}(\Omega) \Rightarrow H_{+}$is linear continuous $(\gamma$ is the trace operator) such that $\gamma u=$ restriction of $u \cdot \bar{n}$ to $\Gamma$ for every $u \in W_{2,0}^{1}(\Omega)$. It is obvious that $\gamma u \in H_{+}$.

Let us introduce the operator $C_{0}=\gamma Q$. In the works $[3,4,5]$ was proved that operator $C_{0}: H_{-} \Rightarrow H_{+}$is positive and compact in the space $H_{0}$.

Let us realize in (3.1) the change of variable for formula $v=A_{1}^{-1 / 2} \xi, \varphi=C_{0}^{-1 / 2} \eta$. Then from (3.1) we get

$$
\begin{aligned}
& \xi=-\nu^{-1} \frac{d}{d t} A_{1}^{-1} \xi+\nu^{-1} V^{*} \eta+\frac{1}{\rho \nu} A_{1}^{-1 / 2} f \\
& -\frac{d \eta}{d t}=\rho g C_{0} V \xi+\delta(x) \frac{d^{2}}{d t^{2}} C_{0} V \xi
\end{aligned}
$$

Here the operators $V=C_{0}^{-1 / 2} \gamma A_{1}^{-1 / 2}: \tilde{L}_{2}(\Omega) \Rightarrow H_{0}$ and $V^{*}=A_{1}^{1 / 2} Q C_{0}^{-1 / 2}: H_{0} \Rightarrow \tilde{L}_{2}(\Omega)$ are bounded $[3,5]$. It is easy to check that $V V^{*}=I: H_{0} \Rightarrow H_{0}$. Then it follows that Ker $V^{*}=\phi$.

Indeed, we have:

$$
(\xi, \xi)_{H_{0}}=\left(V V^{*} \xi, \xi\right)_{H_{0}}=\left(V^{*} \xi, V^{*} \xi\right)_{\tilde{L}_{2}(n)} \quad \forall \xi \in H_{0}
$$

so $V^{*} \xi=0$ only in the case $\xi=0$.

Let us differentiate the first equation of (3.2) with respect to $t$, then from (3.2) we get the following equation

$$
\frac{d \xi}{d t}=-\left(\nu^{-1} A_{1}^{-1}+\nu^{-1} \delta(x) A_{0}\right) \frac{d^{2} \xi}{d t^{2}}-\rho g \nu^{-1} A_{0} \xi+\frac{1}{\rho \nu} A_{1}^{-1 / 2} f_{t}^{\prime}
$$

Here the operator $A_{0}=V^{*} C_{0} V$ is compact in the space $\widetilde{L}_{2}(\Omega)$.

Let us denote the operator $B=\nu^{-1} A_{1}^{-1}+\nu^{-1} \delta(x) A_{0}$. It is obvious that the operator $B$ is positive and compact.

Let us denote $\hat{\xi}=B \xi$. Replacing $\xi$ in the equation $(3,3)$ by $\hat{\xi}$ we get

$$
\frac{d^{2} \hat{\xi}}{d t^{2}}=-B^{-1} \cdot \frac{d \hat{\xi}}{d t}-\rho g \nu^{-1} C \hat{\xi}+\frac{1}{\rho \nu} A_{1}^{-1 / 2} f_{t}^{\prime}
$$

Where $C=A_{0} B^{-1}$ and the operator $-B^{-1}$ is definite negative. It is obvious that the operator $-C\left(B^{-1}\right)^{-1}=-A_{0} B^{-1} B=-A_{0}=-A_{0} B B^{-1}$ is compact, nonpositive and perfectly subordiInates to the operator $B^{-1}$.

- The initial condition of equation (3.4) is written as follows:

$$
\hat{\xi}(0)=B A_{1}^{1 / 2} v^{0}, \quad \hat{\xi}_{t}^{\prime}(0)=B A_{1}^{1 / 2} v^{1}
$$

Therefore, using the theorem 3.3 of the work [6] we obtain that the equation (3.4) with the initial condition (3.5) gets the unique solution $\hat{\xi}$ for every $\left(\hat{\xi}(0), \hat{\xi}_{t}^{\prime}(0)\right) \in B \tilde{L}_{2}(\Omega) \times B \widetilde{L}_{2}(\Omega)$ and $f_{t}^{t} \in C\left(\tilde{L}_{3}(\Omega),[0, T]\right)$. So, for the problem (1.3) - (1.8) we get the following theorem:

Theorem 1. Let there be given $v^{0}(x) \in W_{2,0}^{1}(\Omega), v^{\mathrm{l}}(x) \in W_{2,0}^{1}(\Omega), f(x, \dot{t}) \in C^{1}\left(\tilde{L}_{2}(\Omega),[0, T]\right)$, $\left.p^{0}(x)\right|_{\Gamma} \in H_{-}$there exists the unique solution $v(x, t) \in W_{2,0}^{1}(\Omega)$ of the problem (1.3) - (1.8). 


\section{ON THE SPECTRUM STRUCTURE AND THE COMPLETENESS OF CHARACTERISTIC VECTOR SYSTEM}

In this paragraph we 'll study the equation system (3.2) in the case when the vector function $f(x, t)=0$. Writting the solution of this equation system as $\xi(x, t)=\exp (-\lambda t) \xi(x)$ and $\eta(x, t)=$ $\exp (-\lambda t) \eta(x)$ we get

$$
\xi=\nu^{-1} \lambda A_{1}^{-1} \xi+\nu^{-1} V^{*} \eta ; \quad \lambda \eta=\rho g C_{0} V \xi+\delta(x) \lambda^{2} C_{0} V \xi
$$

It is easy to check that if $\lambda=0$ in the system $(4.1)$ then $(\xi, \eta)=(0,0)$. Indeed, if $\lambda=0$ it follows from (4.1) that

$$
\xi=\nu^{-1} V^{*} \eta ; \quad C_{0} V \xi=0
$$

But in the previous the operator $C_{0}$ is positive in the space $H_{0}$ and $V V^{*}=I: H_{0} \Rightarrow H_{0}$, it follows from (4.2), that $\nu^{-1} \eta=V \xi=0$ and $\xi=0$.

Therefore, zero isn't the characteristic value of the system (4.1) we get the following equation

$$
\lambda I \xi-\lambda^{2}\left(\nu^{-1} A_{1}^{-1}+\delta \nu^{-1} A_{0}\right) \xi-\rho g \nu^{-1} A_{0} \xi=0
$$

or

$$
L(\lambda) \xi \equiv \lambda I \xi-\lambda^{2} B \xi-\rho g \nu^{-1} A_{0} \xi=0
$$

The characteristic values of $L(\lambda)$ coincide with the one of the bundle $L_{1}(\lambda)=I-\lambda B-\rho g \nu^{-1} \lambda^{-1} A_{0}$ In the case when Re $\lambda<0$, using the properties of the operators $B$ and $A_{0}$ we have

$$
\operatorname{Re}\left(L_{3}(\lambda) \xi, \xi\right)_{\tilde{L}_{3}(\Omega)}=(\xi, \xi)_{\tilde{L}_{3}(n)}-\operatorname{Re} \lambda(B \xi, \xi)_{\tilde{L}_{3}(\Omega)}-\frac{\operatorname{Re} \lambda}{\sqrt{\lambda !}}\left(A_{0} \xi, \xi\right)_{\tilde{L}_{2}(\Omega)}>(\xi, \xi)_{\tilde{L}_{3}(\Omega)} \geq 0
$$

It follows that the real parts of the characteristic values $\lambda_{k}(k=1, \infty)$ of the bunde $L_{1}(\lambda)$ or of $L(\lambda)$ from (4.3) are positive. It shows that the motions of the fluid with flotation on the free surface are stable.

Since the operator $B$ and $A_{0}$ are compact, using the results of the work $|13|$ we get the characteristic values $\lambda_{k}$ of the bundle $L(\lambda)$ are discrete and get finite algebraic multiplinity.

If $|\lambda|>\rho g \nu^{-1}|| A_{0} \|$ the bundle $L(\lambda)$ may be written as follows

$$
L(\lambda)=\lambda\left(I-\lambda^{-1} \rho g \nu^{-1} \cdot A_{0}\right) F_{1}(\lambda)
$$

where

$$
\begin{aligned}
& F_{1}(\lambda)=i-\rho g \nu^{-1} A_{n} B-\lambda B-G_{1}(\lambda), \\
& G_{1}(\lambda)=\sum_{n=2}^{\infty} \lambda^{-n+1} \nu^{-n} \rho^{n} g^{n} A_{0}^{n} B \text { and }\left\|G_{1}(\lambda)\right\| \Rightarrow 0 \text { as }|\lambda| \Rightarrow \infty
\end{aligned}
$$

Simce in the case when $|\lambda|>\rho g \nu^{-1} \| A 0$ the operator $I-\lambda^{-1} \rho g \nu^{-1} A_{0}$ is reversible, we consider instead of $L(\lambda)$ the bundle $F_{I}(\lambda)$. The pperator $A_{0}=V^{*} C_{0} V=V^{*} C_{0} V\left(A_{\lambda}^{-1}\right)^{*}$ is compact and $A_{0} A_{1}^{1 / 2}=A_{1}^{1 / 2} Q \Gamma: W_{2,0}^{1}(\Omega) \Rightarrow \tilde{L}_{2}(\Omega)$ is unbounded. Using the definition of the work $[6]$ we get that the operator $A_{0}$ periectly subordinates to the operator $A_{3}$ with the order which is less than. $1 / 2$. Hence, using the consequence $9.4[7, \mathrm{p} .56]$ and the results of the works $[3,8,9]$ we get the following estimation: 


$$
N(r, B)=N\left(r, \nu^{-1} A_{1}^{-1}+\rho g \nu^{-1} A_{0}\right) \cong N\left(r, \nu^{-1} A_{1}^{-1}\right)=c_{1} r^{3 / 2}[1+o(1)] \text { as } r \Rightarrow \infty
$$

where $N(r, B)$ and $N\left(r, \nu^{-1} A_{1}^{-1}\right)$ are the spectrum distribution functions of the operators $B$ and $\nu^{-1} A_{1}^{-1}$ in the region $|\lambda|<r$ and $c_{1}=\nu^{-3 / 2}$ mes $\Omega /(3 \pi)^{2}[8]$.

Therefore, using the theorem 1 of the work [9] and the formula (4.4) we get

$$
N(r, L(\lambda))=N\left(r, F_{1}(\lambda)\right)=N\left(r, \nu^{-1} A_{1}^{-1}\right)[1+o(1)]=c_{1} r^{3 / 2}[1+o(1)] \text { as } r \Rightarrow \infty
$$

Because of the characteristic values $\lambda_{k}$ of the operator $\nu^{-1} A_{1}$ get the limit at infinity and from (4.5), the characteristic values $\lambda_{k}$ of the bundle $L(\lambda)$ get the limit at infinity.

Since the operator $B$ is positive and compact the operator $A_{0} B$ is compact and $\left\|G_{1}(\lambda)\right\| \Rightarrow 0$ as $|\lambda| \Rightarrow \infty$, using the formula (4.5) and the results of the work [10, Theorem 1] we get that the characteristic and associative vector system of $F_{1}(\lambda)$ or either of $L(\lambda)$ in the case when $|\lambda|>$ $\rho g \nu^{-1}\left\|A_{0}\right\|$ is complete in the space $\widetilde{L}_{2}(\Omega)$ excepting the finite defect.

Theorem 2. The characteristic values $\lambda_{k}(k=1, \infty)$ of the bundle $L(\lambda)$ are discrete, get a finite algebraic multiplicity and the limit at infinity and the following estimation is satisfied

$$
N(r, L(\lambda)) \cong N\left(r, \nu^{-1} A_{1}^{-1}\right)=c_{1} r^{3 / 2}[1+o(1)] \quad \text { as }|\lambda| \Rightarrow \infty
$$

In the case when $|\lambda|>\rho g \nu^{-1}\left\|A_{0}\right\|$ the characteristic and associative vector system is complete in $\tilde{L}_{2}(\Omega)$ excepting the finite defect.

If $|\lambda|<\left\|B^{-1}\right\|$ the bundle $L(\lambda)$ may be written as follows:

$$
L(\lambda)=\lambda(I-\lambda B) F_{2}(\lambda)
$$

where

$$
\begin{aligned}
& F_{2}(\lambda)=I-\rho g \nu^{-1} B A_{0}-\rho g \nu^{-1} \lambda^{-1} A_{0}-G_{2}(\lambda) A_{0}, \\
& G_{2}(\lambda)=\sum_{n=2}^{\infty} \lambda^{n-1} \rho g \nu^{-1} B^{n} \text { is compact and }\left\|G_{2}(\lambda)\right\| \Rightarrow 0 \text { as }|\lambda| \Rightarrow 0 .
\end{aligned}
$$

In the work $[5]$ the orthogonal projectors $P_{1}=V^{*} V$ onto $J_{1}(\Omega)$ and $P_{2}=I-P_{1}$ onto $J_{0}(\Omega)$ were produced. It is clear that $\operatorname{Ker} V=J_{0}(\Omega)$ and $P_{2} V^{*}=P_{2} V^{*} V V^{*}=P_{2} P_{1} V^{*}=0$.

At first we consider the operator $A_{0}=V^{*} C V$. After operating two sides of the equation $\left(I-\lambda V^{*} C_{0} V\right) \xi=0$ by the operators $P_{1}$ and $P_{2}$ we get

$$
P_{1} \xi-\lambda P_{1} V^{*} C_{0} V\left(P_{1}+P_{2}\right) \xi=0, \quad P_{2} \xi=0
$$

So we get $P_{1} \xi-\lambda V^{*} C_{0} V P_{1} \xi=0$ or $F_{3}(\lambda) P_{1} \xi \equiv V^{*}\left(I-\lambda C_{0}\right) V P_{1} \xi=0$. But in the previous $\operatorname{Ker} V^{*}=\phi \operatorname{Ker} V=J_{0}(\Omega)$ and $P_{1} \xi \in J_{1}(\Omega)$, the characteristic values $\lambda_{k}$ of the operator $C_{0}$ coincide with the characteristic values of the bundle $F_{3}(\lambda)=V^{*}\left(I-\lambda C_{0}\right) V$ or either with the one of the operator $A_{0}=V^{*} C_{0} V$.

Hence, using $[8]$ we get

$$
N\left(r, \rho g \nu^{-1} C_{0}\right)=N\left(r, \rho g \nu^{-1} A_{0}\right)=c_{2} r^{2}[1+o(1)] \text { as } r \Rightarrow \infty
$$

where $c_{2}=\frac{\nu^{2} \text { mes } \Gamma}{\rho^{2} g^{2} 16 \pi}$.

After operating two sides of the equation $F_{2}(\lambda) \xi=0$ by the operators $P_{1}$ and $P_{2}$ we get 


$$
\begin{aligned}
& P_{1} \xi-\rho g \nu^{-1} P_{1} B A_{0} P_{1} \xi-\rho g \nu^{-1} \lambda^{-1} P_{1} A_{0} P_{1} \xi-P_{1} G_{2}(\lambda) A_{0} P_{1} \xi=0 \\
& P_{2} \xi=\rho g \nu^{-1} P_{2} B A_{0} P_{1} \xi+P_{2} G_{2}(\lambda) A_{0} P_{1} \xi
\end{aligned}
$$

It follows from (4.7) that the characteristic values $\lambda_{k}$ of the bundle $F_{2}(\lambda)$ are coincided with the characteristic values of the bundle $F_{4}(\lambda)=P_{1}-\rho g \nu^{-1} P_{1} B A_{0} P_{1}-\rho g \nu^{-1} \lambda^{-1} P_{1} A_{0} P_{1}-$ $P_{1} G_{2}(\lambda) A_{0} P_{1}$. Since the operator $A_{0}=P_{1} A_{0} P_{1}$ is positive in the space $J_{1}(\Omega)$, using the results of the work $[9$, theorem 1$]$ and the formula (4.6) we get the following estimation

$$
N(r, L(\lambda))=N\left(r, F_{4}(\lambda)\right)=N\left(r^{-1}, \rho g \nu^{-1} A_{0}\right)[1+o(1)]=c_{2} r^{-2}[1+o(1)] \text { as } r \Rightarrow 0
$$

Because of the characteristic values $\lambda_{k}^{-1}$ of the operator $\rho g \nu^{-1} A_{0}$ get the limit at 0 , using the formula (4.8) we obtain that the characteristic values $\lambda_{k}$ of $L(\lambda)$ get the limit at 0 . Using the theorem 1 of the work [10] and the formula (4.7) we get that the characteristic and associative vector system $\left\{P_{1} \xi_{k j}\right\}_{k=1, \infty}^{j=1, m_{k}}$ of the bundle $F_{4}(\lambda)$ or either of $L(\lambda)$ in the case when $|\lambda|<\|B\|^{-1}$ is complete in $J_{1}(\Omega)$ excepting the finite defect.

Theorem 9. The characteristic values $\lambda_{k}$ of the bundle $L(\lambda)$ get the limit at 0 and the following estimation is satisfied

$$
N(r, L(\lambda)) \cong N\left(r^{-1}, \rho g \nu^{-1} C_{0}\right)=c_{2} r^{-2}[1+o(1)] \text { as } r \Rightarrow 0 .
$$

In the case when $|\lambda|<\|B\|^{-1}$ the characteristic ans associative vector system $\left\{P_{1} \xi_{k}\right\}_{k=1, \infty}^{j=1, m_{k}}$ is complete in the space $J_{1}(\Omega)$ excepting finite defect.

In the case when the coefficient of the kinematic viscosity $\nu$ is sufficiently big so that the estimation $\rho g \nu^{-1}\left\|A_{0}\right\|\|B\|<1 / 4$ is satisfied, we introduce the following regions

$$
\begin{aligned}
& \text { 1) } G_{1}=\left\{\lambda: \lambda<\left[1-\Delta_{1}^{1 / 2}\right]\|B\|^{-1 / 2}, \quad \lambda>0\right\} \\
& \text { 2) } G_{2}=\left\{\lambda: \lambda>\left[1+\Delta_{1}^{1 / 2}\right]\|B\|^{-1 / 2}, \quad \lambda>0\right\}
\end{aligned}
$$

where

$$
\Delta_{1}=1-4 \rho g \nu^{-1}\left\|A_{0}\right\|\|B\|
$$

Using the methods of the works $[7,14]$ we easily get the following result

Theorem 4. If the coefficient of kinematic viscosity $\nu$ satisfies the estimation $\rho g \nu^{-1}\left\|A_{0}\right\|\|B\|$ $<1 / 4$, the characteristic values $\lambda_{k}$ are positive and only belong to the regions $G_{1}, G_{2}$ and the problem (4.1) gets the following properties

a) In the region $G_{1}$ the characteristic values $\lambda_{k}$ get limit at 0 and the following estimation is satisfied

$$
N(r, L(\lambda)) \cong N\left(r^{-1}, \rho g \nu^{-1} C_{0}\right)=c_{2} r^{-2}[1+o(1)] \text { as } r \Rightarrow 0,
$$

the characteristic vectors $\left\{P_{1} \xi_{k}\right\}_{k=1}^{\infty}$ are complete in the space $J_{1}(\Omega)$ and haven't got any associative vector and construct the Riss basic of their linear shell.

b) In the region $G_{2}$ the characteristic values $\lambda_{k}$ get limit at $\infty$ and the following estimation is satisfied

$$
N(r, L(\lambda)) \cong N\left(r, \nu^{-1} A_{1}^{-1}\right)=c_{1} r^{3 / 2}[1+o(1)] \text { as } r \Rightarrow \infty,
$$

the characteristic vectors $\left\{\xi_{k}\right\}_{k=1}^{\infty}$ are complete in the space $\tilde{L}_{2}(\Omega)$ and haven't got any associative vector. 


\section{REFERENCES}

1. Gabov S. A. On the problem of ideal fiuid with the flotation. J. of Differential equation. T. 23, No 1, 1987. p. 16-21.

2. Sreteenskii L. N. Theory of wave motions of fluids. Moscov 1977.

3. Kepachevskii N. D., Krein S. G., Ngo Huy Can. Operator methods in Hydrodinamics. EvoIution and spectral problems. 1989, Science, Moscow (In Lisusian).

4. Krein S. G., Lapchev G. I. On the problem of viscous fuid motion in an open vessel. J. of Function analysis and its supplements. T. 2, No 1, 1968 (in Russian).

5. Kopachevskii N. D. On small motion and normal vibration of heavy viscons rotatag fluid systern. Preprint 33-37. FTINT AN USSR. Kharcov, 1970. 60 p.

6. Krein S. G. Linear differential equations in the Banach's space. Science, Moscow, 1966.

7. Markus A. S. Tntroduction to the spectrum theory of polynomial operator bundies. Kishinhev. Stinsa, 1986 (in Russian).

8. Suslina T. A. On the spectrum asymptote of some problems, connecting with the fluid vibations. VINTTI. Leningrad, No 8058 - B85, 1985.

9. Radievskii G. V. Asymptotic distribution of characteristic values of operator-functions analytic in an angle. J. Math. Collection. T. 112 , No 3,1980, p. 395-420.

10. Radievskii G. V. Multiple completeness of Keldys bundle of vectors. J. Math. Collection. T. 91, No 3,1973, p. 310-333.

11. Ladyzhenskaya $O$. A. The mathematical theory of viscous incompressible How. Gordon and Breach, New York, English translation, 1969.

12. Berezanskii Iu. M. Decomposition by characteristic functions of self-adjoint operators. Kiev, Science, Dumḱa, 1985, 800 p.

13. Goxberg I. X., Krein M. G. Introduction to the theory of linear non-self-adjoint operators. Moscow, Science 1965.

14. Askerov N. K., Krein S. G., Laphev G. X. On the problem of viscous fuid vitrations and ids connecting operator equation. Function analysis and its supplements, T. 2, No 2, 1968, p. $22-31$.

15. Tran Thu Ha. On the motion of an ialea! fudid contaired in a elastic bottom vessel with Rotation on free surface. Proceedings of NCSL of Vietnam, Vol. $4,1992$.

Received March 7,1992

\section{VEे CHUYỂ DÔNG CỦA CHÁT LỎNG NHỚT KHÔNG NÉN ĐƯƠC CÓ VẬT NỔI TREÂ MẠT THOÁNG}

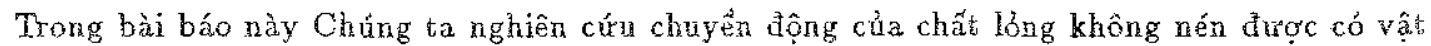
nổ țên mặt thoáng. Đã chưng mimh tính chất tôn tại duy nhất nghiện; nghiên cứu cấu trúc phổ và tín chẩ đầy của hệ yector riêng. 